\title{
Generation of harmonics and sub-harmonics from an internal tide in a uniformly stratified fluid: numerical and laboratory experiments
}

\author{
Ivane Pairaud ${ }^{\mathrm{a}, \mathrm{b},{ }^{*},}$, Chantal Staquet ${ }^{\mathrm{a},{ }^{*}}$, Joël Sommeria ${ }^{\mathrm{a},{ }^{*}}$ and Mahdi M. Mahdizadeh ${ }^{\mathrm{a}, \mathrm{c},{ }^{*}}$ \\ ${ }^{a}$ LEGI, CNRS \& Université de Grenoble, France \\ ${ }^{\mathrm{b}}$ Present address: IFREMER, La Seyne sur Mer, France, \\ ${ }^{c}$ Present address: Hormozgan University, Bandar Abbas, Iran,
}

\author{
*: Corresponding authors : Ivane Pairaud, email address : Ivane.Pairaud@ifremer.fr , Chantal Staquet, email \\ address : Chantal.Staquet@hmg.inpg.fr , Joël Sommeria, email address : Sommeria@coriolis-legi.org , \\ Mahdi M. Mahdizadeh, email address : mehdizadeh@hormozgan.ac.ir
}

\begin{abstract}
:
This paper focuses on the internal tide emitted from a continental slope in a uniformly stratified fluid. Results from numerical simulations using the MITgcm and from laboratory experiments performed on the Coriolis platform in Grenoble are compared. Due to their peculiar dispersion relation, internal gravity waves organize into localized beams of energy. We show that the beam structure is wellpredicted by the viscous theory of [10], assuming that the internal gravity wave field is emitted by a horizontally oscillating cylinder whose radius is the radius of curvature of the topography at the beam emission. The wave beam can bear a sub-harmonic parametric instability whose vertical scale is recovered from resonant interaction theory. Reflection of the wave beam on the bottom leads to the generation of harmonic beams, consisting of free and trapped waves.
\end{abstract}

\section{Introduction}

The oceanic tide can be considered as a barotropic (vertically uniform) oscillating current. In the presence of density stratification, the upward motion induced by bottom topography generates an internal tide. This mechanism is active at mid-ocean ridges and at continental slopes (such as the Bay of Biscay), as documented from satellite altimetry and moored current meter data. Internal tide has been given much 
attention for the last ten years in relation with their role in deep ocean mixing, as reviewed by [3]. The magnitude and distribution of mixing indeed influences the whole process of oceanic thermohaline circulation ([2]). Mixing is produced from the internal tide by the degradation of the internal tide into turbulence through a sequence of instabilities, involving resonant interactions (such as parametric instability $[8,17,4])$ or shear or buoyancy induced instabilities.

Several laboratory experiments of internal wave generation by oscillating bodies have been performed since the pioneering work of [18]. The case of a horizontally oscillating cylinder has been recently studied by [25] as a simple model of internal tide generation. Indeed, in a frame of reference attached to the barotropic tide, the topography oscillates horizontally and the active part of the topography may be modeled as a cylinder. [19] considered an isolated topography while the case of a continental slope was addressed by [1], [6] and [21].

The numerical modelling of the internal tide first relied on the hydrostatic approximation and solved the equations of motions in an oceanic context (f.i. [9]). To our knowledge, the first non-hydrostatic numerical simulation of internal tide emission was performed by [12] with the MITgcm, focusing upon energy transfer from the barotropic tide to internal tides for an isolated topography. Further works were conducted along the same lines by [13] and [14] for the same topography. The case of a continental slope was considered by [5] and [4]. All these numerical works were performed in a two-dimensional vertical plane and for an idealized topography. The case of a three-dimensional realistic topography with non-hydrostatic equations was tackled by [11].

In the present paper, the internal tide is generated by a simple two-dimensional continental slope in a uniformly stratified ocean and modeled by joint numerical simulations and laboratory experiments. Their set-ups are described in Sect. 2. The internal tide emission is discussed and illustrated from our results in Sect. 3. The main features of the internal tide are presented in the next sections: (i) its spatial structure close to the emission region is well predicted by a viscous theoretical model of internal wave emission by a horizontally oscillating cylinder (Sect. 4), (ii) it can bear a sub-harmonic instability of small vertical scale (Sect. 5), (iii) harmonic components can be generated from the bottom reflexion region (Sect. 6). We conclude in Sect. 7.

\section{Experimental set-ups}

\subsection{Laboratory experiments}

A tidal current is forced along a channel, 4 meter wide, built inside the circular tank of the Coriolis platform, 13 meters in diameter (see Fig. 1). The results of the present paper are obtained in the absence of rotation. The barotropic tide is produced by the horizontal oscillation of a piston, filling the whole section of the channel. At the 
opposite end, the channel is open, so that the flow can escape side-way over the topography. Let $d$ and $\omega$ be the amplitude and frequency of the motion of the piston respectively. In the present paper, the values for $d$ and $2 \pi / \omega$ are $0.6 \mathrm{~cm}$ and $26 \mathrm{~s}$ respectively, except in Sect. 5 where $d=2 \mathrm{~cm}$.

A linear density stratification is introduced by salinity while the tank is filled. Since there is no flux of salinity through the surface and through the bottom of the tank, the linear density profile is slowly eroded by mixed layers developing from these boundaries. Nevertheless their thickness remains limited to a few $\mathrm{cm}$ during a whole set of experiments (several weeks) with no significant evolution of the stratification in the interior. The data presented here correspond to a stratification with uniform Brunt-Väisälä frequency $N_{\text {exp }}=0.71 \pm 0.2 \mathrm{rad} / \mathrm{s}^{-1}$, except for a bottom mixed layer $\simeq 9 \mathrm{~cm}$ thick and a surface mixed layer $\simeq 3 \mathrm{~cm}$ thick.

The laboratory configuration is designed by similarity with a typical oceanic configuration, as described in the numerical simulations of the internal tide by [5]. To reach regimes of inertial dynamics, with weak viscous damping, we need a sufficiently deep water, so we choose a total depth of $H=90 \mathrm{~cm}$, with a shelf height equal to $76.5 \mathrm{~cm}$. The topography is made of a constant slope 0.5 , with inclination angle $30^{\circ}$, matched with the flat continental shelf through a section with radius of curvature $R=180 \mathrm{~cm}$. If this is assumed to represent an ocean $4.5 \mathrm{~km}$ deep, the vertical scaling factor is $1 / 5000$. At this scale, the available channel length of $10 \mathrm{~m}$ represents $50 \mathrm{~km}$. To simulate the process over a distance of $250 \mathrm{~km}$, allowing space for internal tide propagation, reflection and breaking, we need to apply a distortion of aspect ratio by a factor 5 . Hence, the slope is five times larger in the experiment than in the ocean (typical value 0.1 ). We expect that such a distortion has only a weak influence on the dynamics. Note that within the hydrostatic approximation, the dynamics is strictly invariant by a change of aspect ratio in the absence of viscosity effect or turbulence parameterisation.

The main measurement tool is Particle Image Velocimetry (PIV) providing velocity fields in a vertical plane. Polystyrene particles (300 $\mu \mathrm{m}$ in diameter) sorted in density are used to get a uniform seeding over the fluid depth. The vertical laser sheet is obtained by a 6 watt continuous Yag laser and an oscillating mirror while the images are obtained by two CCD cameras looking through windows on the side

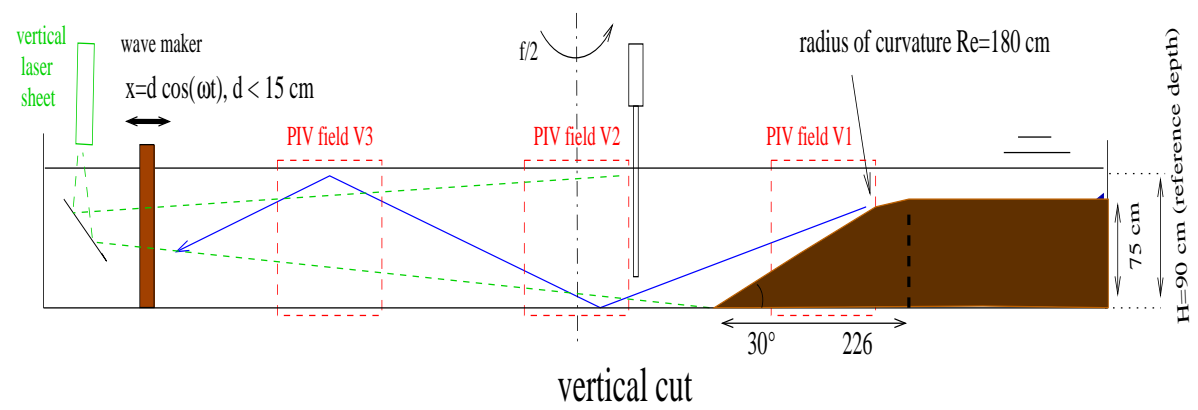

Fig. 1 Sketch of the laboratory experiment. 
of the channel. A few velocity fields have been also obtained from a top view with a horizontal laser plane.

\subsection{Numerical simulations}

The simulations are based on the numerical model developed at MIT by [16], which we adapted to the experimental configuration. The code solves the nonlinear nonhydrostatic Navier-Stokes equations in the Boussinesq approximation using a finite volume method and a Cartesian coordinate system. Since the topography in the laboratory experiment is two-dimensional and the Coriolis platform is non rotating, we assume that the flow dynamics is two-dimensional in a vertical plane. The horizontal and vertical dimensions of the numerical domain are exactly those of the laboratory experiment as is also the topography we impose. The coordinate system $(O, x, z)$ we use is such that $x$ increases from the shelf to the piston and $z=0$ at the bottom. The position of $O$ along the $x$-axis is located at the crossing of the line $z=76.5 \mathrm{~cm}$ (on the shelf) and the line of maximum slope tangent to the topography.

We performed direct numerical simulations that is, no subgrid scale modelling is used. The value of the viscosity is set to $10^{-2} \mathrm{~cm}^{2} / \mathrm{s}$, as in the laboratory experiments, while the Prandtl number is set to 1 (against 700 in the experiments).

The MITgcm has an implicit free-surface formulation and no-flux boundary conditions are applied to the density field at the surface and at the bottom. Free-slip boundary conditions are applied to the velocity components.

We chose $N=0.72 \mathrm{rad} / \mathrm{s}$ in the simulation which we impose from the free surface down to a height $z=9 \mathrm{~cm}$, below which the density is uniform. Thus, we ignored the thin surface mixed layer of the experiments but reproduced the bottom mixed layer.

The forcing generated by the piston is modeled by imposing a barotropic flux at the two vertical boundaries. This flux is oscillating in time at the excitation frequency $\omega$. Measurements of the barotropic component in the experiment indicate that it is partly blocked by the topography, so that the forcing amplitude decreases from the piston to the shelf-break by typically $50 \%$. The constant barotropic flux introduced in the computation is therefore adjusted to match the measured value over the topography, in the region of internal tide generation. Thus, in the numerical simulation, $d=0.24 \mathrm{~cm}$ except in Sect. 5 where $d=0.5 \mathrm{~cm}$.

We use a resolution of 1202 grid points along the horizontal direction, with $d x=0.91 \mathrm{~cm}$. Along the vertical, the grid size is constant and equal to $d z=0.28$ $\mathrm{cm}$, implying that the number of grid points is 320 over the plain and 48 over the shelf. This resolution may be considered as large since, in an ocean model, it would correspond to a horizontal resolution of $45 \mathrm{~m}$ and to a vertical direction of $14 \mathrm{~m}$. One tidal period is simulated by 200 time steps, with $d t=0.1305 \mathrm{~s}$.

All simulations are started from rest and run over 40 forcing periods. 


\section{Emission of the wave beam}

Internal gravity waves are dispersive waves with an anisotropic dispersion relation (f.i. [15]). For a plane wave in a stratified medium with constant $N$, the wave vector $\mathbf{k}$ and frequency $\omega$ are indeed related by $\omega^{2}=N^{2} \sin ^{2} \theta$, where $\theta$ is the angle of $\mathbf{k}$ with the vertical. The group velocity is perpendicular to $\mathbf{k}$, therefore aligned with the fluid velocity. As a consequence, any object oscillating at a frequency $\omega$ smaller than $N$ emits internal gravity waves, which carry the energy along directions with angle $\theta$ with respect to the horizontal. Energy is concentrated along these directions within wave beams. This striking property is nicely illustrated by the laboratory experiments of [18]. The experiments also show that the beams are tangent to the object as a consequence of the impermeability condition. Beams are thus emitted from 'critical' points, where the boundary is perpendicular to k. The slope is referred to as being 'critical' there. In the case of a cylinder, the energy of the source distributes among four beams, associated with upward and downward (as well as leftward and rightward) propagation.

The theory of wave emission by a cylinder has been derived in the far-field by [24] and later in the near-field by [10]. In the latter, the structure of the wave beam depends both upon the ratio $s / R$, where $s$ is the distance along the beam from the object and $R$ the radius of the cylinder, and on the ratio $\delta / R$, where $\delta=\sqrt{v / \omega}$ is the thickness of the viscous boundary layer on the object. It is assumed that $\delta$ is much smaller than $R$. In this case, near the cylinder, the energy of each beam actually possesses two maxima, which eventually merge into a single one away from the cylinder under the action of viscosity. For a very large cylinder, the two maxima are well pronounced, which results in two distinct wave beams on each side of the object.

The internal tide can be viewed as the wave emission from an horizontally oscillating topography, in a frame of reference attached to the barotropic tide. In the case of a continental slope, the energy will be able to propagate in two directions only. This result is illustrated in Fig. 2 where color maps of the along-beam velocity are displayed for the laboratory experiment (frame a) and for the numerical simulation (frame b). Our study is focused on the beam propagating toward the deep ocean, and the beam propagating leftward (toward the shelf) is not shown in these figures.

A good agreement is observed between the simulation and the experiment, the emission location, thickness and amplitude of the wave beams being the same. Note that, already after 8 periods, the maximum amplitude of the velocity field is $1 \mathrm{~cm} / \mathrm{s}$ that is, at least three times larger than the forcing amplitude.

Fig. 2 shows that the most active part of the topography is the region where the beam is tangent to the slope that is at the critical point. Hence, we may compare our results for wave emission to the theoretical predictions of [10] for a cylinder with radius $\mathrm{R}=1.80$ tangent to the slope at the critical point. Such a comparison was also made by [25], [19] and [6] using data from their laboratory experiments. 


\section{Spatial structure of the wave beam}

In the theory of [10], the linear Boussinesq equations of motions are solved in a two-dimensional vertical plane and for a constant $N$ fluid, for a solution which is harmonic in time. The key assumptions of the model lie in the boundary conditions for the velocity field. This field vanishes at infinity, as expected. On the cylinder, the thickness of the viscous boundary layer $\delta$ is assumed to be much smaller than the cylinder radius so that free-slip boundary conditions can be set. As a result, an analytic expression for the stream function can be obtained, from which the along-beam velocity field $U_{H K}$ can be inferred. Let $s$ and $\eta$ be the along- and cross-beam coordinates respectively. Omitting time harmonic dependence, $U_{H K}(s, \eta)$ is expressed as

$$
U_{H K}(s, \eta)=-\frac{U_{0}}{2} e^{-i \theta} \int_{0}^{+\infty} \mathrm{J}_{1}(k) \exp \left(k^{3} \lambda \frac{s}{R}+i k \frac{\eta}{R}\right) d k,
$$

where $U_{0}$ is the velocity amplitude of the cylinder, $\lambda=(\tan \theta / 2)(\delta / R)^{2}$ and $\mathrm{J}_{1}(k)$ is the first order Bessel function.

In the present work, we compute in the absolute frame of reference the velocity filtered at the forcing frequency, which therefore involves the baroclinic and barotropic tidal signals. The corresponding analytical expression of the along-beam velocity component, denoted $U_{s}$, is therefore (omitting again time dependence)

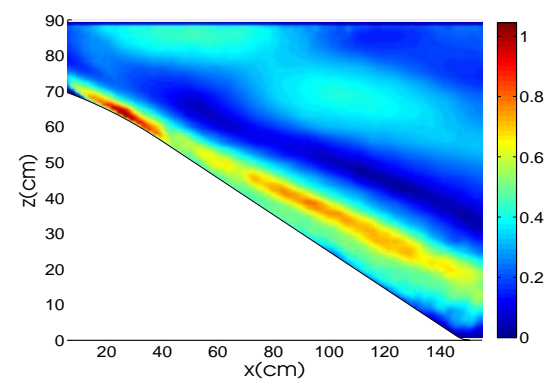

(a)

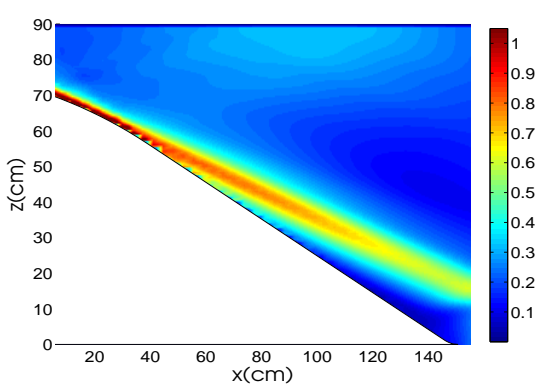

(b)

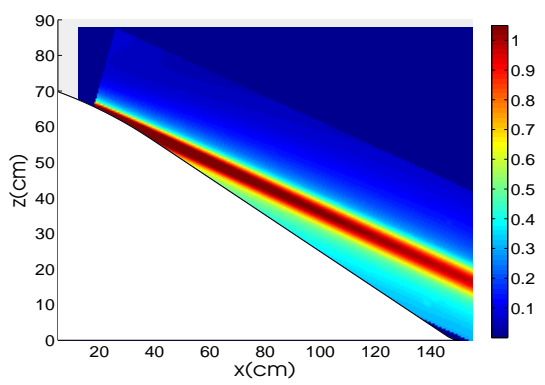

(c)

Fig. 2 (a) Laboratory experiment. Spatial distribution of the amplitude of the along-beam velocity component (in $\mathrm{cm} / \mathrm{s}$ ) filtered at the tidal frequency and averaged over the 7 th and 8th tidal periods; (b) Same as (a) for the numerical simulation. (c) Theoretical prediction from [10] using equation (2), for a horizontal cylinder oscillating horizontally with radius equal to the radius of curvature of the topography at the critical slope. 


$$
U_{s}(s, \eta)=-U_{H K}(s, \eta)+U_{B T},
$$

where $U_{B T}$ is the barotropic component of the velocity, which depends upon $x . U_{B T}$ is computed as the vertical average of the horizontal velocity component. When used in equation (2), the factor $U_{0}$ in equation (1) is taken as the amplitude of the barotropic velocity at the critical point.

Maps of $U_{s}$ are plotted in Fig. 2c. The topography has been added to facilitate comparison with the results from the numerical simulations and laboratory experiments (but we recall that waves are emitted by a horizontally oscillating cylinder in the theory). The agreement between the three approaches is quite good, the beam amplitudes differing by $20 \%$ at most.

\section{Parametric instability of the wave beam}

It has been known since [7] and [20] that plane monochromatic internal gravity waves may be unstable through parametric subharmonic instability (PSI). This instability is a special case of resonant interactions which occur among a wave triad. Let $\left(\mathbf{k}_{0}, \omega_{0}\right),\left(\mathbf{k}_{1}, \omega_{1}\right)$ and $\left(\mathbf{k}_{2}, \omega_{2}\right)$ denote the wave vector and frequency of each member of the triad. Since the waves form a triad, $\mathbf{k}_{0}+\mathbf{k}_{1}+\mathbf{k}_{2}=0$. Resonance occurs when a temporal resonance relation is also satisfied : $\omega_{0}+\omega_{1}+\omega_{2}=0$. In the case of PSI, one wave of small amplitude, referred to as the primary wave, interacts with two waves of much smaller amplitude such that $\left|\omega_{1}\right| \simeq\left|\omega_{2}\right| \simeq\left|\omega_{0}\right| / 2$, where index 0 refers to the primary wave. The instability promotes the growth of small scale waves with respect to the primary wave scale.

As discussed above, the internal tide is emitted as a wave beam, which can be viewed as a plane wave whose amplitude is modulated across the beam ([22]). This implies that a wave beam of infinite extent is a solution of the Boussinesq equations in the absence of viscosity, as is a plane wave in an infinite medium. Hence, such a wave beam could bear a PSI. This was shown indeed by [4] from numerical simulations of internal tide emission at a continental slope in an oceanic context.

In the present case of joint numerical and laboratory experiments, no PSI was detectable for the forcing amplitude used in Fig. 2. Since the growth rate of PSI is proportional to the amplitude of the wave beam, we ran a computation doubling the forcing amplitude. The result is displayed in Fig. 3a, where we plot the time average amplitude of the horizontal velocity component filtered at half the forcing frequency. Quasi-horizontal structures (actually whose direction makes the angle $\operatorname{asin}(\omega / 2 N)$ with the horizontal) appear. These structures are of alternate sign along the vertical (it is not visible since the amplitude of the velocity field is plotted). The same spatial pattern was obtained by [4]. We performed this filtering operation on the experimental data, for the same forcing amplitude (frame $3 \mathrm{~b}$ ): similar quasihorizontal structures appear, with comparable amplitude, demonstrating for the first time in a laboratory experiment that PSI can appear in a wave beam. Note that the 
maximum amplitude of the perturbation is only 4 times smaller than the maximum amplitude of the wave beam from which the perturbation developed.

The spatial structure of this perturbation displays a well-defined vertical scale, which can be computed from resonant interaction theory. Fig. 3c displays the growth rate of the instability as a function of the vertical component of the wave vector $k_{z}$, for the parameters of the computation. In the absence of viscous effects, the growth rate saturates as $k_{z} \rightarrow \infty$ so that viscous effects, in damping the largest wavenumbers, introduce a scale selection. The value of $k_{z}$ for which the growth rate is maximum should provide the scale of the instability visible in Fig. 3a and $3 \mathrm{~b}$. We find from Fig. $3 \mathrm{c}$ that this vertical wavelength is about $8 \mathrm{~cm}$, which compares well with that of the quasi-horizontal structures displayed in frames a) and b). This result confirms that the instability is of parametric sub-harmonic type.

\section{Generation of harmonics}

When the wave beam hits the bottom mixed layer, a reflected beam is created. While no harmonic wave is generated by the reflection of a plane internal gravity wave on a flat bottom, this result does not hold any longer for a wave beam ([23]). This

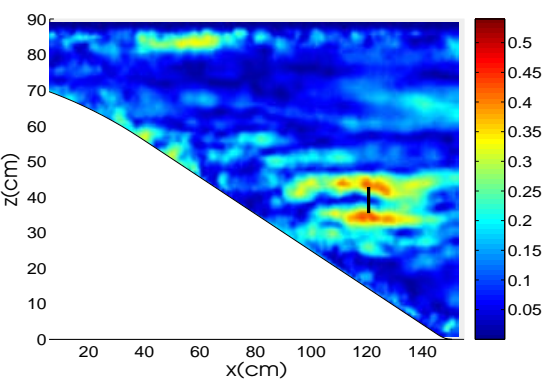

(a)

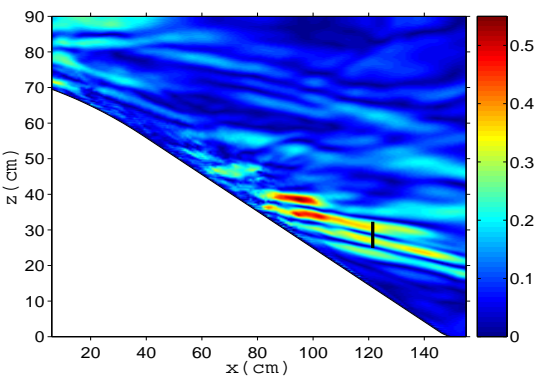

(b)

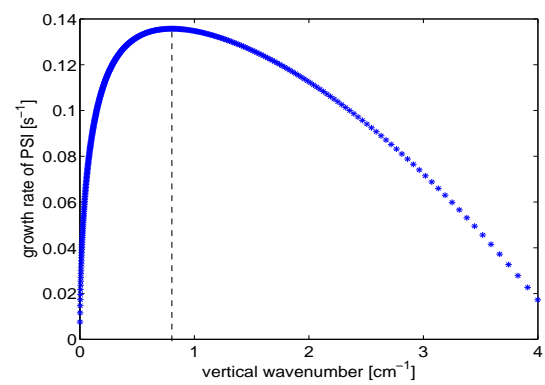

(c)

Fig. 3 (a) Laboratory experiment. Amplitude of the horizontal velocity (in $\mathrm{cm} / \mathrm{s}$ ) filtered at half the forcing frequency and averaged over forcing periods 22 to 25; (b) Same as (a) for the numerical simulation. (c) Growth rate of PSI predicted by resonant interaction theory for the parameters of the simulation and experiment, as a function of the vertical wavenumber of the perturbation. The vertical wavenumber with maximum growth rate is indicated with a dashed line and the corresponding wave length is drawn on frames (a) and (b) with a vertical black line. 
theoretical prediction was verified by [5] and is also found in the present numerical simulation, as shown in Fig. 4b. This figure displays the time average amplitude of the horizontal velocity component filtered at twice the forcing frequency (which is smaller than $N$ ). The harmonic wave reflects also at the top boundary of the mixed layer and on the piston, which accounts for the perturbed signal for $x$ larger than about $4 \mathrm{~m}$. Fig. 4a shows that a similar harmonic wave is created in the laboratory experiment. It is noteworthy that, both in the laboratory and numerical experiments, a harmonic wave is also trapped in the primary reflected wave beam.

\section{Conclusion}

The purpose of this short paper was to provide the main characteristics of the weakly nonlinear dynamics of the internal tide emitted at a continental slope in a uniformly stratified non rotating fluid. These are the spatial structure of the wave beam and two processes of energy transfer toward smaller scales. Large scale laboratory experiments were performed on the Coriolis platform, so that inertial dynamics can be reached, and compared with well-resolved numerical simulations using the non hydrostatic MITgcm for the same sets of parameters.

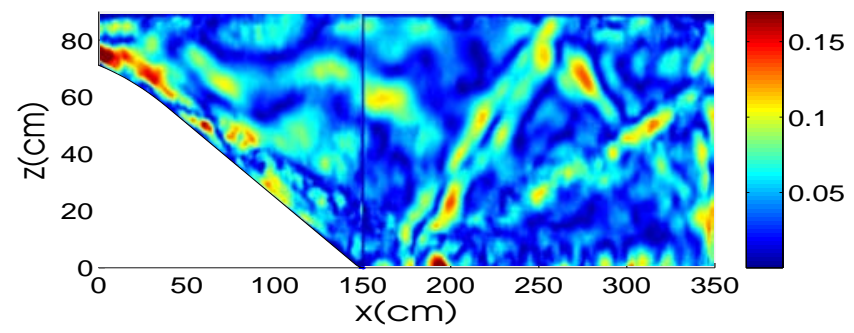

(a)

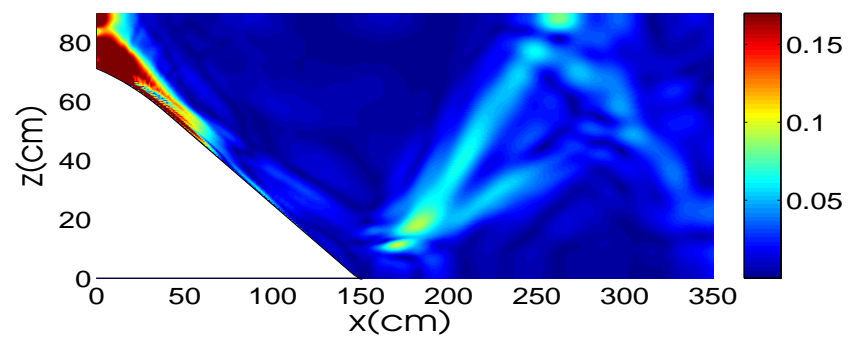

(b)

Fig. 4 (a) Laboratory experiment. Amplitude of the horizontal velocity component filtered at twice the forcing frequency and averaged over forcing periods 18 to 21; (b) Same as (a) for the numerical simulation. 
We analysed the emission of the wave beam in light of the theory of [10], when the oscillating source is a cylinder with radius equal to the radius of curvature of the topography at the critical slope. A very good agreement is found between the laboratory and numerical experiments and the theory, with differences not larger than $20 \%$. This shows that the radius of curvature of the topography at the critical slope, along with viscosity, control the beam formation and possibly the beam width. The theory of [10] should provide such dependencies.

The beam can be unstable to parametric sub-harmonic instability (PSI) if the time scale of the instability (namely, the inverse growth rate) is smaller than the viscous time at the scale of the instability. A PSI was observed both in the laboratory and in the numerical experiments, associated with strikingly quasi-horizontal phase lines of comparable amplitude. We checked that the vertical wavelength of the perturbation matches the prediction from resonant interaction theory. When extrapolated to the ocean, using a simple similarity argument, this vertical wavelength is $400 \mathrm{~m}$, associated with layers of half that height. The much higher value of the Reynolds number in the ocean compared to the present experiments would actually select an even smaller scale. If these layers become unstable (through buoyancy induced instability), an appreciable vertical part of the ocean would be mixed.

We also showed that harmonic beams can be created when the incident wave beam interacts with the reflected beam at the bottom mixed layer, a part of the harmonic wave field being trapped in the reflected beam. The same behavior is observed in the numerical simulation and, for the first time again, in the laboratory experiments.

Acknowledgements We thank Louis Gostiaux and Bruno Voisin for fruitful interaction. The laboratory experiments and the post-doctoral grant of Ivane Pairaud were supported by ANR (Agence Nationale de la Recherche) through contract ANR-05-BLAN-0176-01. Numerical simulations were performed on the computers of IDRIS (Institut du Développement et des Ressources en Informatique Scientifique) under contract 070580.

\section{References}

1. Baines, P.G., Fang, X.H.: Internal tide generation at a continental shelf/slope junction: A comparison between theory and a laboratory experiment. Dynamics of Atmospheres and Oceans 9, 297-314 (1985).

2. Ferrari, R., Wunsch, C: Ocean circulation kinetic energy: reservoirs, sources, and sinks. Ann. Rev. Fluid Mech. 41, 253-282 (2009).

3. Garrett, C, Kunze, E.: Internal tide generation in the deep ocean. Annu. Rev. Fluid Mech. 39, 57-87, 2007.

4. Gerkema T., Staquet C., Bouruet-Aubertot, P.: Nonlinear effects in internal tide beams and mixing. Geophysical Research Letters. 33, L08604 (2006).

5. Gerkema T., Staquet C., Bouruet-Aubertot, P.: Nonlinear effects in internal tide beams and mixing. Ocean Modelling 12, 302-318 (2006).

6. Gostiaux, L, Dauxois, T. Laboratory experiments on the generation of internal tidal beams over steep slopes. Physics Fluids 19(1), 1-4 (2007).

7. Hasselmann, K: A criterion for nonlinear wave stability. J. Fluid Mech. 30, 737 (1967). 
8. Hibiya, T., Nagasawa, M., Niwa, N.: Nonlinear energy transfer within the oceanic internal wave spectrum at mid and high latitudes. J. Geophys. Res. 107(C11), 3207 (2002).

9. Holloway, P.E., Merrifield, M.A.: Internal tide generation by seamounts, ridges, and islands. J. Geophys. Res. 104(C11), 25937-25951 (1999).

10. Hurley, D.G., Keady, K.: The generation of internal waves by vibrating elliptic cylinders. Part 2. Approximate viscous solutions. J. Fluid Mech. 351, 11 (1997).

11. Jachec, S.M., Fringer, O.B., Gerritsen, M.G., Street, R.L.: Numerical simulation of internal tides and the resulting energetics within Monterey Bay and the surrounding area. Geophys Res. Lett. 33, L12605 (2006).

12. Khatiwala, S.: Generation of internal tides in an ocean of finite depth: analytical and numerical calculations. Deep Sea Research, 50, 3-21 (2003).

13. Lamb, K.B.: Nonlinear interaction among internal wave beams generated by tidal flow over supercritical topography. Geophys. Res. Lett. 31, doi:10.1029/2003GL01939 (2004).

14. Legg, S., Huijts, K.M.H: Preliminary simulations of internal waves and mixing generated by finite amplitude tidal flow over isolated topography. Deep Sea Research-II, 53,140-156, 2006.

15. Lighthill, J.: Waves in fluids. Cambridge University Press (1978).

16. Marshall, J., Adcroft A., Hill C., Perelman L., Heisey, C: A finite-volume, incompressible navier-stokes model for studies of the ocean on parallel computers. Journ. Geophys. Res. 102:5753-5766 (1997).

17. MacKinnon, J.A., Winters, K.B.: Subtropical Catastrophe: significant loss of low-mode tidal energy at 28.9 degrees. Geophys. Res. Lett. 32, L15605, doi:10.1029/2005GL023376 (2005).

18. Mowbray, D.E., Rarity, B.S.H.: The internal wave pattern produced by a sphere moving vertically in a density stratified liquid. J. Fluid Mech. 30, 489-495 (1967).

19. Peacock, T., Echeverri, P., Balmforth, N.J.: An experimental investigation of internal wave beam generation by two-dimensional topography. J. Phys. Ocean. 38, 235-242 (2008).

20. Phillips, O.M.: The dynamics of the upper ocean. Cambridge University Press (1966).

21. Staquet, C., Sommeria, J., Goswami, K., Mehdizadeh, M.: Propagation of the internal tide from a continental shelf : laboratory and numerical experiments. Proceedings of the Sixth International Symposium on Stratified Flows, Perth, Australia, 11-14 December (2006).

22. Tabaei, A., Akylas, T.R.: Nonlinear Internal Gravity Wave Beams. J. Fluid Mech. 482, 141161 (2003).

23. Tabaei, A., Akylas, T.R., Lamb, K.G.: Nonlinear Effects in Reflecting and Colliding Internal Wave Beams. J. Fluid Mech. 526, 217-243 (2005).

24. Thomas, N.H., Stevenson, T.N.: A similarity solution for viscous internal waves. J. Fluid Mech. 54, 495-506 (1972).

25. Zhang, H.P., King, B., Swinney, H.L.: Experimental study of internal gravity wvaes genarated by supercritical topography. Physics Fluids bf 19, 096602 (2007). 